\title{
Anti-CD105 Inhibits Primary Cancer Growth and Secondary Hematoge- nous Metastasis in a Xenograft Model
}

\author{
Rahmawati Minhajat, Li Hou, Keita Kai, Yukari Takase, Daisuke Mori and Osamu Tokunaga*
}

Department of Pathology and Biodefense, Faculty of Medicine, Saga University, Japan

\begin{abstract}
CD105, a receptor for TGF-b1 and -b-3 modulates TGF-b signaling by interacting with TGF-bRI and/or II. It is now emerging as a prime vascular target of antiangiogenetic cancer therapy.

In the present study, we investigate the efficacy of CD105 in colorectal cancer models. We found decreases in tumor size and in the number of metastatic foci to lung in a xenograft cancer model in scid mouse that was treated with Anti-CD105 antibody. The necrotic area in the tumor was greater in the anti-CD105 antibody group than that of the control group. The number of metastatic foci and the area of metastasis were also lesser in the anti-CD105 group than those of the control group. A direct effect of anti-CD105 antibody to the cultured colon cancer cell line was not detected in respect to morphology and proliferation. Decreased vasculature by an antiangiogenic effect of anti-CD105 antibody was confirmed by an immunohistochemical assessment of CD105 expression on frozen tumor tissue.

These findings demonstrated that CD105 was specifically expressed in vascular endothelial cells in a xenograft colon cancer model, and anti-CD-105 antibody inhibited both tumor growth and hematogenous metastasis by blocking the vascular network. CD105 is a useful target and anti-CD105 antibody is a candidate for antiangiogenic colorectal cancer therapy.
\end{abstract}

Keywords: CD105, endoglin, angiogenesis, anti-angiogenesis therapy, scid mouse, colon cancer.

\section{INTRODUCTION}

Angiogenesis is an important step in the process of cancer growth [1]. It is a multistep process and promotes metastasis involving the spread from the primary tumor through blood and lymphatic vessels and subsequent growth of the secondary tumor at the anchored distant organs. The established theory on the most potent mechanism of angiogenesis is based on the interaction between vascular endothelial growth factor (VEGF) and the receptor (VEGF-R). To block the VEGF activity, the first generation drugs that were designed to bind to VEGF have been developed [2] and the new second generation drugs were designed to block the VEGF action through inactivation of the tyrosine kinases of VEGF-R [3,4]. These drugs have shown a promising result in cancer growth inhibition and in a reduced death rate in some cancer types. Because the drug's targeting is tyrosine kinase of VEGF-R, which distributes in the vasculature of any tissue, it will require additional careful and long term follow up study on the side effects.

CD105, a receptor for TGF-b1 and -b3, modulates TGF-b signaling by interacting with TGF-bRI and/or II [5]. CD105 is predominantly expressed on activated endothelial cells and its promoter is strongly and selectively active in endothelial cells $[6,7]$. Elevated levels of CD105 expression were consistently detected on human microvascular endothelium and

*Address correspondence to this author at the Department of Pathology and Biodefense, Saga University, Faculty of Medicine, 5-1-1 Nabeshima, Saga 849-8501, Japan; Tel: 81-952-34-2230; Fax: 81-952-34-2055;

E-mail: tokunao@cc.saga-u.ac.jp on vascular endothelial cells in tissues undergoing active angiogenesis, such as inflamed tissues and tumors. We previously reported that CD105 was expressed exclusively in the de novo blood vessels of colon cancer, and its expression is related to the depth of invasion and lymph node metastasis [8]. Furthermore, our previous studies have shown that CD105 was expressed specifically in the tumor angiogenesis of brain, lung, breast, stomach, and colon cancers [9]. Takahashi N. et al. reported that the systemic administration of naked antihuman Endoglin mAb can suppress established tumors, and the efficacy is markedly enhanced by combining a chemotherapeutic drug using an antiangiogenic schedule of drug dosing [10].

In the present study, we investigated the efficacy of CD105 to the colon cancer model in the SCID mouse.

\section{MATERIALS AND METHODS:}

\section{Cells and Proliferation Assay}

The cancer cell line, WiDr, was obtained from the American type culture collection (ATCC, Rockville). The cancer cells were grown in RPMI-1640 medium (Nissui Pharmaceutical Co., Tokyo), supplemented with 5\% fetal bovine serum (FBS, Life Technologies, Gaithersburg), $2 \mathrm{mM}$ L-glutamine (GIBCO, Invitrogen Co., NY) and 1.5 g/L Sodium Bicarbonate (Wako Pure Chemical Industries Ltd., Osaka), 50mg/ml gentamicin sulfate (Schering Plough, Osaka) and maintained in an incubator with saturated humidity and $5 \% \mathrm{CO} 2$ at $37^{\circ} \mathrm{C}$. The cells were seeded and grown in a monolayer in $25 \mathrm{~cm} 2$ culture flasks (Iwaki Glass, Chiba). The cells were harvested from the exponential 
growth phase by a brief trypsin treatment. The cell concentration was determined by a hemocytometer.

For the proliferation assay, the cancer cells were cultured in a chamber slide $\mathrm{T}^{\mathrm{TM}}$ (Nalgen Nunc International, NY) for 6 $\mathrm{h}$, and then incubated with anti-CD105 antibody for $24 \mathrm{~h}$. After fixation with cold acetone, the cells were reacted with a monoclonal mouse anti-human Ki67 antigen (MIB-1, DakoCytomation, Denmark) at a dilution 1:50. The sections were incubated overnight at $4^{\circ} \mathrm{C}$, washed, and then incubated with the secondary antibody goat anti-mouse IgG (EnVision, DakoCytomation) for $30 \mathrm{~min}$ at room temperature. The positive staining of Ki67 in the nuclei was counted from 10 high power field areas.

\section{Cancer Lesion Development in Back Skin and Lung of the SCID Mouse}

Twenty three 5-week-old male SCID mice C.B-17/Icr (20-25 g) purchased from Clea Co. (Tokyo) were used in this study. All mice were maintained under specific pathogenfree conditions at the Center for Animal Experimentation, Saga University School of Medicine (Saga, Japan), in accordance with the Guide for Animal Experimentation, Saga University School of Medicine, and Japanese Governmental Law 105, which requires us to undertake experiments using the minimal number of animals. All mice were given a standard chow and water ad libitum. The cancer cells were prepared from exponentially growing cells that were harvested with brief treatment of $0.25 \%$ tryspin- 0.01 EDTA solution and resuspended in $\mathrm{PBS}$ at a final concentration of $3 \times 10^{7}$ cells per ml. $1 \times 10^{7}$ cells per mouse was injected subcutis in the back (SC group, $n=13$ ) and tail vein (Hematogenous group, $\mathrm{n}=11)$. The anti-angiogenesis experiment was initiated 4 weeks after the cancer cell implantation when the tumors developed to a size of $20-25 \mathrm{~mm}$ in diameter. Treatment for the SC group was subdivided into the following two groups: untreated control group $(n=3)$ and intra peritoneal injection group with anti-mouse CD105 antibody (MJ7/18, monoclonal, (Santa Cruz Biotechnology, CA, USA) $(n=4)$ at $50 \mu \mathrm{g} /$ every 2 days. The treatment of lung metastatic tumor were the untreated control group $(n=3)$ and the anti-CD105 antibody group $(n=8)$. The injected doses were the same as those of the subcutaneous tumor group. During the treatment, the mice were monitored daily for tumor growth and morbidity. The body weight and the tumor size were measured once a week. The tumor size was calculated using the following formula: Size=length $\mathrm{x}$ width, and expressed as $\mathrm{mm}^{2}$. For evaluation of necrotic areas in sc group and of tumor areas in the lung metastasis, HE stained tissue sections were scanned and then analyzed using the NIH image softwareprogram. They each were counted using the following formula and expressed as \%: combined necrotic areas / total section area, and the tumor areas/whole section areas of lung, respectively.

\section{Immunohistochemical Staining of CD-105 Positive Vas- culature in Cancer}

The subcutaneous tumor tissue was frozen immediately after the sacrifice. It was cut with a cryostat at $6 \mu$ thickness, and fixed with cold acetone. The sections were incubated with primary antibody Rat-anti mouse CD105 monoclonal antibody diluted at 1:100 (Chemicon International,
CA,USA), overnight at $4^{\circ} \mathrm{C}$, washed, and then incubated with secondary antibody Rabbit Anti-Rat IgG (DakoCytomation), for $30 \mathrm{~min}$ at room temperature. The CD105 positive blood vessels were counted and expressed as the average from 5 areas of hot spots, expressed in the average, and expressed as the microvessel density (MVD).

\section{Statistic Analysis}

The significance of the differences in tumor size, body weight, necrotic areas in xenograft tissue, tumor area in lung metastasis, the degree of positive expression of Ki67, and the vascular endothelial expression of CD105 were examined by Student's $t$ - test. $P<0.05$ was considered significant.

\section{RESULTS}

\section{No Effect on Proliferation of Colon Cancer Cell Line by Anti-CD105 Antibody}

The colon cancer cell line WiDr was originally established from moderately differentiated adenocarcinoma (ATCC). They grow in a monolayer when cultured in a dish. The effect of Anti-CD105 antibody on cell proliferation was immunocytochemically determined by the expression of Ki67. A slight decrease in Ki-67 positive cells was seen, but no significant difference was found between the control and the Anti-CD105 antibody group, 153.8 vs 128.6. We decided to use the WiDr cell line for the xenograft colon cancer model in SCID mice.

\section{Inhibitory Effect on Xenograft Cancer Model}

Four weeks after the cultured colon cancer cells were implanted in the subcutis of the back, the tumor developed nodules (Fig. (1A)) and the size increased with time (Fig. (1B)). We compared the tumor size after treatment with antiCD105. The size of tumor declined from $163.6 \mathrm{~mm}$ to 131.6 $\mathrm{mm}$ after treatment for 2 weeks with anti-CD105 antibody (Fig. (1C)), and it remained unchanged for 1 week thereafter. The tumor size, however, was unchanged or even gradually regrew until sacrifice despite the same treatment regimen with anti-CD105 antibody as before. Whereas, the tumor size of the control group increased from $171.2 \pm 13.6 \mathrm{~mm}^{2}$ to $180.5 \pm 63.2 \mathrm{~mm}^{2}$, the size continued growing up to 4 weeks of sacrifice (Fig. (1B)). The body weight of the mice with the s.c. xenograft and the lung metastasis group decreased from an average of $23.2 \pm 0.8$ and $22.3 \pm 3.5$ grams to $21.8 \pm 2.8$ and $21.1 \pm 3.7$ grams, respectively, after treatment with AntiCD105. The body weight was unchanged and even decreased after 4 weeks of implantation (Table 1). This resulted from the cachexia of the individual mouse bearing cancer in the back or lung. The necrotic areas of the colon cancer xenograft are shown in Fig. (2A). Necrosis tended to occur in the center, but the margin of the cancer lesion was well preserved. In the control mouse, the cancer lesion grew in solid and necrosis was hard to see. But as the cancer lesion grew, a patchy distribution of necrotic foci was observed at the end of the experiment. Each focus was smaller than that of the anti-CD105 antibody group (Fig. (2B)). A significant difference between the control and the Anti-CD105 group was found $(20.8 \pm 8.4 \%$ vs. $53.5 \pm 11.0 \%$, p<0.05) (Fig. $(\mathbf{2 C}))$. 


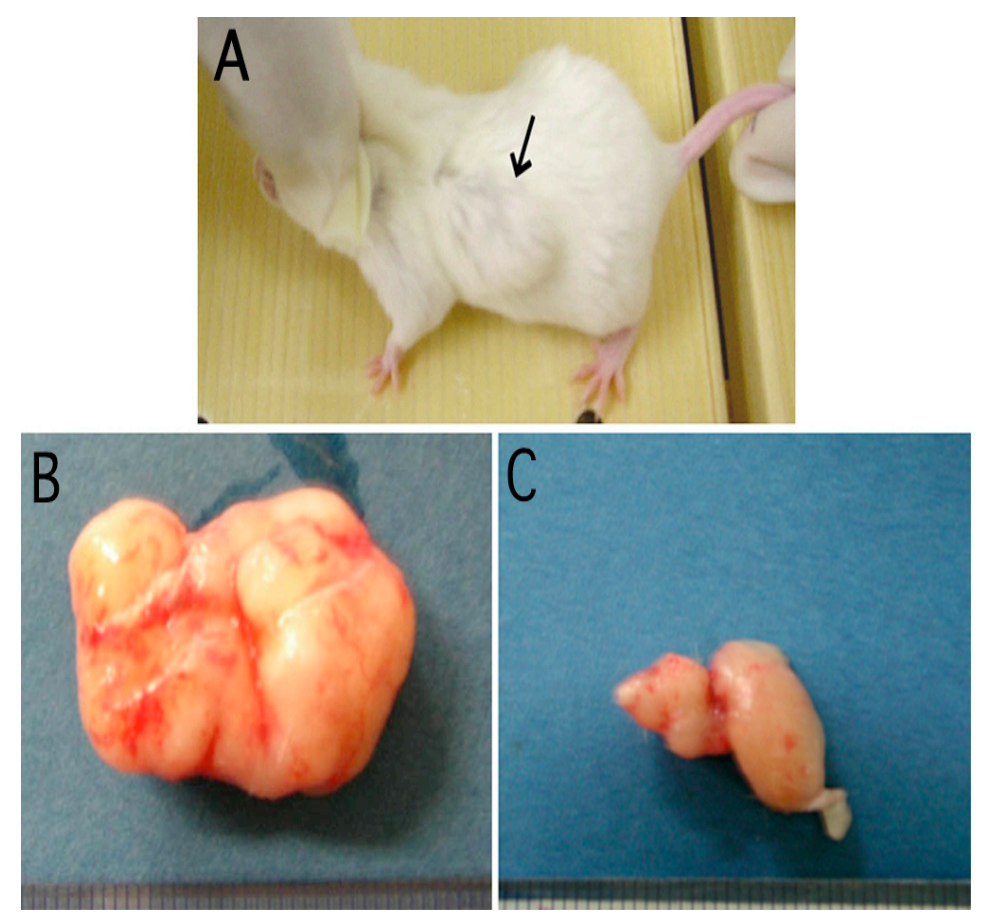

Fig. (1). Cancer development in the SCID mouse. A) Subcutaneous implantation of the colon cancer cells forms a cancer nodule in the subcutis (arrow). B) An enucleated cancer nodule is large 8 weeks after the implantation (Control). C) The cancer nodule shrunk after each 2-day administration of anti-CD105 antibody for 4 weeks.

Table 1. A Summary of the Body Weight and Tumor Status in Both the Subcutaneous Implantation Group and the Hematogenous Metastatic Cancer Group

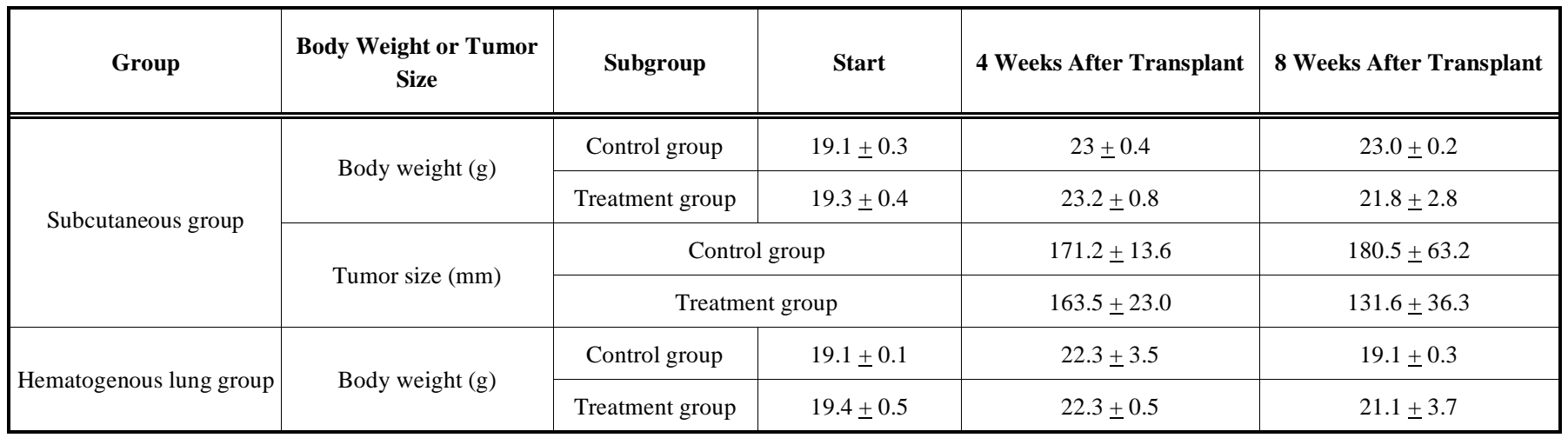

\section{Expression of CD105 in Xenograft Cancer Lesion}

The blood vessels in the cancer lesions were stained by anti-CD105 antibody and the positive vessels were counted as MVD. The vascular network was stained sharply in a slender configuration reflecting a well organized vasculature in the control (Fig. (3A)). In the anti-CD105 antibody group, on the other hand, the continuity of the capillary network was interrupted everywhere in the cancer tissue and the cancer cells were easy to degenerate due to the lack of a blood supply. The remaining capillaries proved to be fragmented and degenerative when immunostained by anti-CD105 antibody. The survived endothelial cells were stained individually (Fig. (3B)). Therefore, the antiangiogenic effect of antiCD105 was confirmed by immunohistochemistry on the fro- zen cancer tissue. The result was significantly different between the control and the anti-CD105 antibody group $(211 \pm 11.1$ vs $63.0 \pm 8.4 p<0.01$ (Fig. (3C)).

\section{Hematogenous Cancer Metastasis in Lung}

The cancer cells administered to the tail vein traveled to the lung in the blood flow and anchored on the capillary beds there. In the control group, many foci of cancer lesions were seen. They grew into large nodules as the time passed (Fig. $(\mathbf{4 A}, \mathbf{C}))$. On the other hand, the number of cancer metastases is much lesser and the size is also smaller (Fig. (4B)). Significant differentiation between the control and the AntiCD105 antibody was found $(2.25 \%$ vs $0.09 \%, p<0.01)$ (Fig. (4D)). 

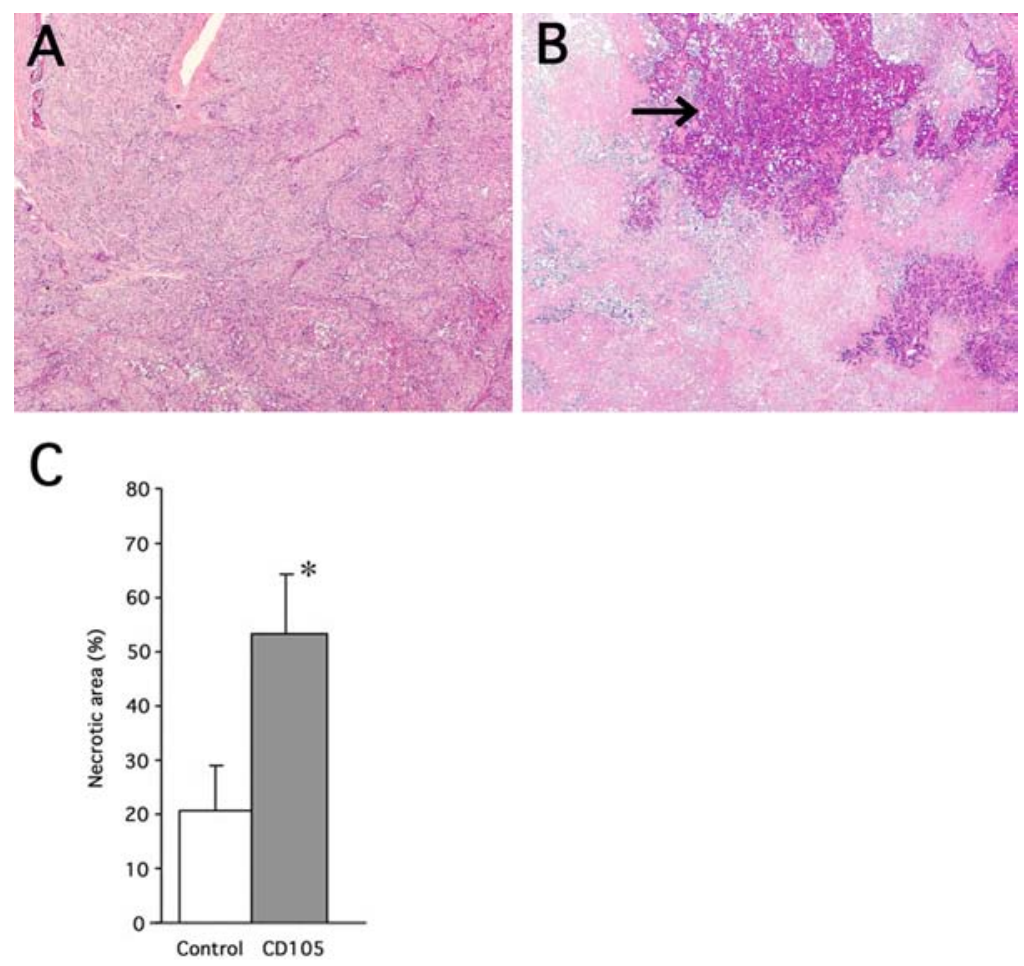

Fig. (2). Effect of anti-CD105 antibody administration. A) Necrosis of the cancer tissue is scanty in the control. $x 40$. B) Necrotic area occupies almost half of the cancer lesion by anti-CD105 antibody treatment. Red islands are the remaining cancer tissue (arrow). $\mathrm{x} 40$. C) Relative necrotic area in the whole cancer lesion (\%). Effect on the cancer necrosis is much greater in the anti-CD105 antibody group. $* P<0.05$.
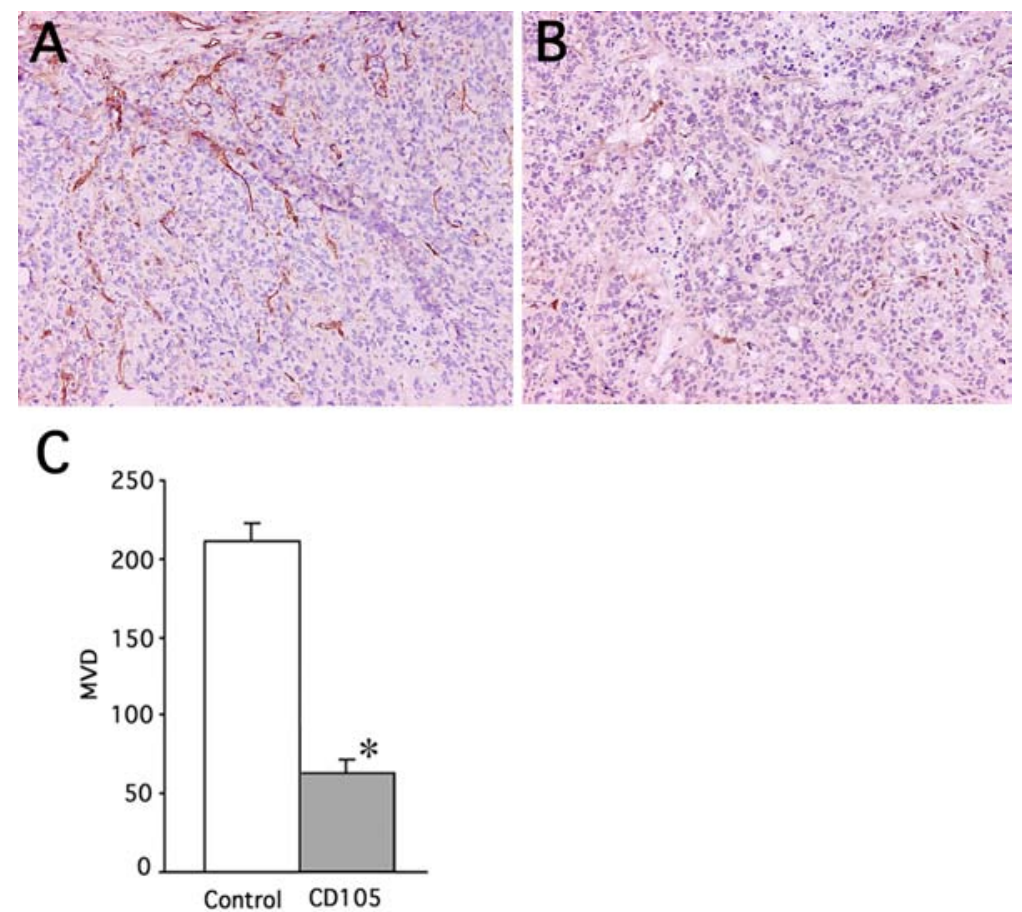

Fig. (3). An immunohistological study of CD105 expression and MVD in the subcutaneous cancer lesion. A) The vascular network is well developed and CD105 is strongly expressed in the control. x100 B) The vascular network is often interrupted and the remaining fragmented vasculature is barely positive. Note the degenerative or necrotic cancer tissue. $x 100$. C) Comparison of MVD. MVD is much less in the antiCD105 group. $* P<0.01$. 


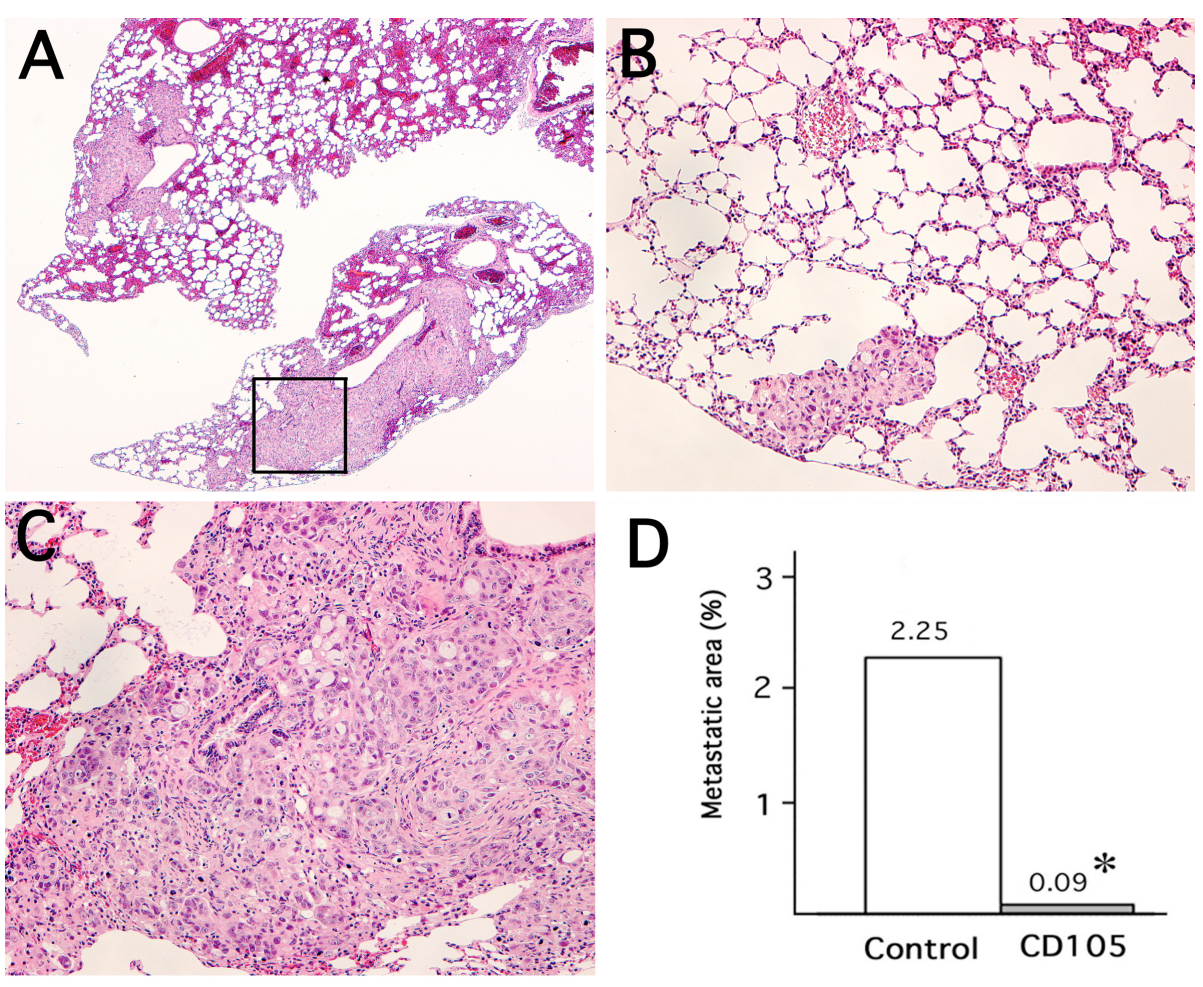

Fig. (4). A comparison of hematogenous metastasis to lung. A) Many foci of metastatic lung cancer are seen in the control group. $x 40$. B) On the other hand, the number of cancer metastases is much smaller and the size is also smaller. x100. C) Higher magnification of A. Most of the cancer lesion is large. $\mathrm{x} 200$. D) A comparison of the cancer metastasis. The number of cancer foci is much less in the anti-CD105 group. * $P<0.01$.

\section{DISCUSSION}

Angiogenesis is an important step in the process of cancer growth [1]. It promotes metastatic spread by providing the means for cells to detach from the primary tumor site, travel in the blood stream, and anchor at the distant metastatic site.

The vascular endothelium of tumor and normal tissue is heterogeneous [11]. Recent advances in immunohistological and biochemical technologies have made it possible to detect the heterogeneous endothelium. Among those markers, vascular endothelial growth factor (VEGF) is a potent mitogen in angiogenesis. It plays a central role in both normal vascular development and tumor neovascularization [12]. All of the VEGF-A isoforms bind to both VEGFR-1 and VEGFR2 .

von Willebrand Factor (vWF) is the most familiar and reliable marker, and has been used for a long time as a conventional endothelial marker, although it is more strongly expressed in larger blood vessels than in capillary endothelial cells. vWF is also expressed in bone marrow megakaryocytes and platelets.

Other more specific reagents, CD31 and CD34, are now considered to be optimal pan-endothelial markers [13]. Though vWF, CD34, and CD31 are used as pan-endothelial markers, they react not only with newly formed blood vessels but also with mature blood vessels. Therefore, the question arises as to whether these pan-endothelial markers are ideal for the evaluation of tumor angiogenesis.
CD105, a receptor for TGF-b1 and -b-3, modulates TGFb signaling by interacting with TGF-bRI and/or II, and it is emerging as a prime vascular target of antiangiogenetic cancer therapy $[14,15]$.

CD105 has been shown to be a proliferation-associated and hypoxia-inducible protein, and is preferentially expressed in the activated endothelial cells participating in neoangiogenesis [6], and is undetectable or weakly expressed in the vessels of normal tissues [8], with a marked tissue specificity [16].

Because the interruption of the blood supply to the tumor results in a reduction of the tumor size, necrosis, or shrinkage of the tumor, CD105 is therefore considered to be a prime vascular target of antiangiogenetic cancer therapy [14].

CD105 has been demonstrated to be a more specific and sensitive marker for tumor angiogenesis than CD31 [17] and CD34 in gastric cancer $[18,19]$.

Anti-angiogenic drugs have been developed for cancer treatment targeting VEGF, VEGF receptors, and PDGF receptors. Specifically, agents that prevent VEGF-A binding to its receptors and antibodies that directly block VEGFR-2 achieved reduction in tumor sizes and improvement in progression-free survival [20]. However, side effects are also observed to occur because such molecules are universally distributed in the human vascular system.

Based on the recent advances in CD105 studies, it is the most specific and sensitive molecule in activated prolifera- 
tive endothelial cells within tumors. Because angiogenesis is crucial for tumor growth, CD105 is becoming a possible target for anti-angiogenesis cancer therapy [21]. It has been shown that anti-CD105 antibodies specifically bind activated endothelial cells in angiogenic tissues [22]. Anti-CD105 monoclonal antibodies (mAb) significantly inhibit the proliferation of cultured human endothelial cells [23].

DNA vaccines directed against CD105 are under trial in animal models. Oral DNA vaccines elicited a CD8+ mediated immune response against the CD105-positive target cells and suppressed dissemination of pulmonary metastasis of breast carcinoma cells [24]. The targeting of CD105, as therapeutic anti-angiogenic therapy in cancer has been extensively investigated in a series of anti-CD105 mAb administration in combination with radiolabeled, immunotoxinconjugated, or conventional chemotherapeutic regimens. Interestingly, in all of these animal models, the anti-tumor efficacy and anti-metastasis activities were identified, and proved the ability of anti-CD105 mAb to inhibit tumorassociated angiogenesis [10]. In our experimental model, anti-CD105 antibody therapy was conducted on a xenograft colon cancer model in SCID mice. The cancer growth ceased and shrunk dramatically due to necrosis in the center of the tumor following intraperitoneal administration of antiCD105 antibody every 2 days. Besides, such anti-CD105 antibody therapy prevented cancer dissemination in the lung when the cancer cells were administered from the tail vein. Such antibody therapy also prevented the secondary metastasis from the primary subcutaneous site of cancer mass developed in the back of the mice.

The use of anti-CD105 antibody for anti-angiogenic therapy has a great advantage in targeting the tumor. If antiCD105 antibody is combined with anticancer chemotherapeutic agents it may therefore become a potent therapeutic tool for cancer in the future, while also being useful in follow-up studies for the reduction or regrowth of cancer.

\section{ACKNOWLEDGEMENT}

The author thanks Mr. F. Mutoh and Mrs. E. Kawasaki for their skillful technical assistance.

\section{REFERENCES}

[1] Folkman J. Tumor angiogenesis:therapeutic implications. N Engl J Med 1971; 285: 1182-6.

[2] Berlin JD, Venook A, Bergsland E, et al. A phase II trial of T138067, a novel microtubule inhibitor, in patients with metastatic, refractory colorectal carcinoma. Clin Colorectal Cancer 2008; 7: 44-7.

[3] Adams VR, Leggas M. Sunitinib malate for the treatment of metastatic renal cell carcinoma andgastro-intestinal stromal tumors. Clin Ther 2007; 29: 1338-53.

[4] Oudard S, George D, Medioni J, Motzer R. Treatment option in renal cell carcinoma: Past and future. Ann Oncol Suppl 2007; 10: $\mathrm{X} 25-31$.
[5] Barbara NP, Wrana JL, Letarte M. Endoglin is an accessory protein that interacts with the signaling receptor complex of multiple members of the transforming growth factor-superfamily. J Biol Chem 1999; 274: 584-94.

[6] Graulich W, Nettelbeck DM, Fischer D, et al. Cell type specificity of the human endoglin promoter. Gene 1999; 227: 55-62.

[7] Miller DW, Graulich W, Karges B, et al. Elvated expression of endoglin, a component of The TGF- $\beta$-receptor complex, correlates with proliferation of tumor endothelial cells. Int J Cancer 1999; 81: 568-72.

[8] Minhajat R, Mori D, Yamasaki F, et al. Endoglin (CD105) expression in angiogenesis of colon cancer: Analysis using tissue microarrays and comparison with other endothelial markers. Virchows Arch 2006; 448: 127-34.

[9] Minhajat R, Mori D, Yamasaki F, et al. Organ-specific endoglin (CD105) expression in the angiogenesis of human cancers. Pathol Int 2006; 56: 717-23.

[10] Takahashi N, Haba A, Matsuno F, et al. Antiangiogenic therapy of established tumor in human skin/severe combined immunodeficiency mouse chimeras by anti-endoglin (CD105) monoclonal antibody, and synergy between anti-endoglin antibody and cyclophosphamide. Cancer Res 2001; 61: 7846-54.

[11] Chi JT, Chang HY, Haraldsen G, et al. Endothelial cell diversity revealed by global expression profiling. Proc Natl Acad Sci 2003; 100: 10623-8.

[12] Ferrara N. VEGF and the quest for tumor angiogenesis factors. Nat Rev Cancer 2002; 2: 795-803.

[13] Vermeulen PB, Gasparini G, Fox SB, et al. Second internationa consensus on the methodology and criteria of evaluation of angiogenesis quantification in solid human tumours. Eur J Cancer 2002; 38: 1564-79.

[14] Fonsatti E, Altomonte M, Nicotra MR, et al. Endoglin (CD105): a powerful therapeutic target on tumor-associated angiogenetic blood vessels. Oncogene 2003; 22: 6557-63.

[15] Sarah E, Duff SE, Chenggang LI, et al. CD105 is important for angiogenesis: evidence and Potential applications. FASEB J 2003; 17: 984-92.

[16] Burrows FJ, Derbyshire EJ, Tazzari PL, et al. Up-regulation of endoglin on vascular endothelial cells in human solid tumors: implications for diagnosis and therapy. Clin Cancer Res 1995; 1: 1623-34.

[17] Behrem S, Zarkovic K, Eskinja N, et al. Endoglin is a better marker than CD31 in evaluation of angiogenesis in glioblastoma. Croat Med J 2005; 46: 417-22.

[18] Ding S, Li C, Lin S, et al. Comparative evaluation of microvessel density determined by CD34 or CD105 in benign and malignant gastric lesions. Hum Pathol 2006; 37: 861-6.

[19] Saito M, Sato Y, Watanabe J, et al. Angiogenic factors in normal endometrium and Endometrial adenocarcinoma. Pathol Int 2007; 57: 140-7.

[20] Marx J. Encouraging results for second-generation antiangiogenesis drugs. Science 2007; 308: 1248-9.

[21] Fonsatti E, Maio M. Highlights on endoglin (CD105): from basic findings towards clinical applications in human cancer. J Transl Med 2004; 2: 18

[22] Taskiran C, Erdem O, Onan A, et al. The prognostic value of endoglin (CD105) expression in ovarian carcinoma. Int J Gynecol Cancer 2006; 16: 1789-93.

[23] Maier JA, Delia D, Thorpe PE, et al. In vitro inhibition of endothelial cell growth by the anti-angiogenic drug AGM-1470[TNP-470] and the anti-endoglin antibodyTEC-11. Anticancer Drugs 1997; 8: 238-44.

[24] Lee SH, Mizutani N, Mizutani M, et al. Endoglin (CD105) is a target for an oral DNA vaccine against breast cancer. Cancer Immunol Immunother 2006; 55: 565-74.

This is an open access article licensed under the terms of the Creative Commons Attribution Non-Commercial License (http://creativecommons.org/licenses/by-nc/3.0/) which permits unrestricted, non-commercial use, distribution and reproduction in any medium, provided the work is properly cited. 